Pacific Journal of Mathematics

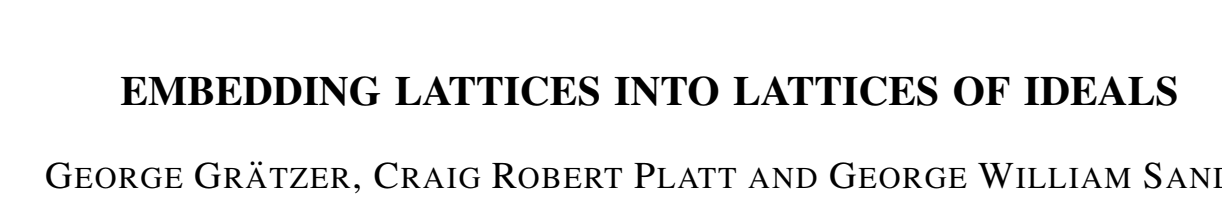




\title{
EMBEDDING LATTICES INTO LATTICES OF IDEALS
}

\author{
G. Grätzer, C. R. Platt, and B. Sands
}

A lattice $L$ is transferable iff, whenever $L$ can be embedded in the ideal lattice of a lattice $M$, then $L$ can be embedded in $M$. This concept was introduced by the first author in 1965 who also proved in 1966 that in a transferable lattice there are no doubly reducible elements. In fact, he proved that every lattice can be embedded in the ideal lattice of a lattice containing no doubly reducible elements. In a recent paper of the first two authors, the idea emerged that one should study transferability via classes $K$ of lattices with the property that every lattice is embeddable in the ideal lattice of a lattice in $K$. This approach was used to establish that transferable lattices are semi-distributive. This investigation is carried further in this paper. Our main result shows that every lattice can be embedded in the ideal lattice of a lattice satisfying the two semi-distributive properties and two variants of Whitman's condition.

1. Introduction. It was shown by G. Grätzer ([6], [7]) that every transferable lattice $L$ satisfies the condition

(X) $L$ has no doubly reducible element.

In fact, he proved a stronger result, namely, that every lattice can be embedded in the ideal lattice of a lattice satisfying $(X)$.

In general, if $(P)$ is a lattice-theoretic property which is preserved by sublattices and which satisfies the assertion

$\mathscr{E}(P)$ : every lattice can be embedded in the ideal lattice of a lattice satisfying $(P)$,

then $(P)$ is a property of all transferable lattices. In addition to $(X)$, properties of a lattice $L$ for which this assertion is known to hold include

$(S F) \quad L$ is sectionally finite (that is, all principal ideals are finite);

$\left(S D_{\wedge}\right)$ for $a, b, c \in L, a \wedge b=a \wedge c$ implies that $a \wedge b=a \wedge(b \vee c)$;

$\left(S D_{\vee}\right)$ for $a, b, c \in L, a \vee b=a \vee c$ implies that $a \vee b=a \vee(b \wedge c)$.

That $\mathscr{E}(S F)$ holds is a consequence of P. M. Whitman's embedding 
theorem [10] and the observation that the partition lattice on a set $S$ is isomorphic to the ideal lattice of the lattice of all finite partitions of $S$; that $\mathscr{E}\left(S D_{\wedge}\right)$ and $\mathscr{E}\left(S D_{\vee}\right)$ hold is the content of a recent paper of G. Grätzer and C. R. Platt [8].

Consider the properties

$(W)$ for $a, b, c, d \in L, a \wedge b \leqq c \vee d$ implies that $[a \wedge b, c \vee d] \cap\{a, b, c, d\} \neq \varnothing$

$\left(W_{l}\right)$ for $a, b, c, d \in L, c \leqq a \wedge b \leqq c \vee d$ implies that $[a \wedge b, c \vee d] \cap\{a, b, c, d\} \neq \varnothing$

$\left(W_{u}\right)$ for $a, b, c, d \in L, a \wedge b \leqq c \vee d \leqq a$ implies that $[a \wedge b, c \vee d] \cap\{a, b, c, d\} \neq \varnothing$.

K. Baker and A. W. Hales [2] proved that if a lattice satisfies $(W)$, then so does its ideal lattice. Hence $\mathscr{E}(W)$ fails; however, in this paper, we will show that $\mathscr{E}\left(W_{l}\right)$ and $\mathscr{E}\left(W_{u}\right)$ hold. In fact, we will prove that every lattice can be embedded in the ideal lattice of a lattice satisfying the four properties $\left(S D_{\vee}\right),\left(S D_{\wedge}\right),\left(W_{l}\right)$, and $\left(W_{u}\right)$ simultaneously. More succinctly, our main result is

THEOREM. $\mathscr{E}\left(\left(S D_{\vee}\right) \&\left(S D_{\wedge}\right) \&\left(W_{l}\right) \&\left(W_{u}\right)\right)$ holds.

It follows from the theorem and the preceding remarks that every transferable lattice is sectionally finite and satisfies $\left(S D_{\vee}\right)$, $\left(S D_{\wedge}\right),\left(W_{l}\right)$, and $\left(W_{u}\right)$. By a result of R. Antonius and I. Rival [1], we conclude:

COROLlaRY. Every transferable lattice satisfies $(W)$.

The proof of the theorem is contained in $\S 2$. In $\S 3$ we shall settle the truth or falsity of $\mathscr{E}(P)$ for most remaining combinations $(P)$ of the above properties. In particular, it will be shown that $\mathscr{E}\left(\left(S D_{\vee}\right) \&(S F) \&(X)\right)$ holds and that $\mathscr{E}\left((S F) \&\left(S D_{\wedge}\right)\right)$ and $\mathscr{E}\left((S F) \&\left(W_{u}\right)\right)$ fail. With these results, we can determine the status of $\mathscr{E}(P)$ for all but two combinations $(P)$ of the properties $(X),(S F),\left(S D_{\vee}\right),\left(S D_{\wedge}\right),\left(W_{l}\right)$, and $\left(W_{u}\right)$. These two will be given at the end of the paper.

\section{Proof of the theorem.}

Definition 1. Let $L$ be a lattice and let $\langle a, b, c, d\rangle$ be an ordered quadruple of elements of $L$. Then we will say that

(i ) $\langle a, b, c, d\rangle$ is a $\left(W_{l}\right)$-failure if $c \leqq a \wedge b \leqq c \vee d$ and $[a \wedge b, c \vee d] \cap\{a, b, c, d\}=\varnothing$; 
(ii) $\langle a, b, c, d\rangle$ is a $\left(W_{u}\right)$-failure if $a \wedge b \leqq c \vee d \leqq a$ and $[a \wedge b, c \vee d] \cap\{a, b, c, d\}=\varnothing$;

(iii) $\langle a, b, c, d\rangle$ is an ( $\left.S D_{\wedge}\right)$-failure if $a \wedge b=a \wedge c=d$ and $a \wedge(b \vee c) \neq d$

(iv) $\langle a, b, c, d\rangle$ is an (SD $)$-failure if $a \vee b=a \vee c=d$ and $a \vee(b \wedge c) \neq d$;

(v) $\langle a, b, c, d\rangle$ is a failure if it is any of the above four types of failures.

Definition 2. Let $L$ be a lattice, let $\langle a, b, c, d\rangle$ be a failure in $L$, and let $\phi$ be a homomorphism from a lattice $M$ onto $L$. Then $\varphi$ repairs $\langle a, b, c, d\rangle$, or $\langle a, b, c, d\rangle$ is repaired in $M$ by $\varphi$, iff $\left\langle a^{\prime}, b^{\prime}, c^{\prime}, d^{\prime}\right\rangle$ is never a failure in $M$ of the same type as $\langle a, b, c, d\rangle$, for any $a^{\prime} \in \varphi^{-1}(a), b^{\prime} \in \varphi^{-1}(b), c^{\prime} \in \varphi^{-1}(c)$, and $d^{\prime} \in \varphi^{-1}(d)$.

Lemma 3. Let $K, L$, and $M$ be lattices, let $\varphi_{1}: M \rightarrow L$ and $\varphi_{2}: L \rightarrow K$ be onto homomorphisms, and let $\langle a, b, c, d\rangle$ be a failure in $K$. If $\langle a, b, c, d\rangle$ is repaived in $L$ by $\varphi_{2}$, then it is repaired in $M$ by $\varphi_{2} \circ \varphi_{1}$.

Proof. Each of the four conditions $\left(S D_{\vee}\right),\left(S D_{\wedge}\right),\left(W_{l}\right)$, and $\left(W_{u}\right)$ can be expressed in the form $P(x, y, z, w) \Rightarrow Q(x, y, z, w)$, where $P$ and $Q$ are disjunctions of polynomial equations and hence are preserved under homomorphisms. Since $\langle a, b, c, d\rangle$ is a failure in $K$, there exist appropriate $P$ and $Q$ such that $P(a, b, c, d)$ holds but $Q(a, b, c, d)$ fails. Suppose that $\langle a, b, c, d\rangle$ is not repaired in $M$ by $\varphi_{2} \circ \varphi_{1}$; then there are elements $a^{\prime}, b^{\prime}, c^{\prime}, d^{\prime} \in M$ such that $\left(\varphi_{2} \circ \varphi_{1}\right)\left(x^{\prime}\right)=x$ for $x \in$ $\{a, b, c, d\}, P\left(a^{\prime}, b^{\prime}, c^{\prime}, d^{\prime}\right)$ holds, and $Q\left(a^{\prime}, b^{\prime}, c^{\prime}, d^{\prime}\right)$ fails. Consequently, $P\left(\varphi_{1}\left(a^{\prime}\right), \varphi_{1}\left(b^{\prime}\right), \varphi_{1}\left(c^{\prime}\right), \varphi_{1}\left(d^{\prime}\right)\right)$ holds in $L$. Since $\langle a, b, c, d\rangle$ is repaired in $L$ by $\varphi_{2}$, this implies that $Q\left(\varphi_{1}\left(a^{\prime}\right), \varphi_{1}\left(b^{\prime}\right), \varphi_{1}\left(c^{\prime}\right), \varphi_{1}\left(d^{\prime}\right)\right)$ holds in $L$. But now $Q(a, b, c, d)=Q\left(\varphi_{2}\left(\varphi_{1}\left(a^{\prime}\right)\right), \varphi_{2}\left(\varphi_{1}\left(b^{\prime}\right)\right), \varphi_{2}\left(\varphi_{1}\left(c^{\prime}\right)\right), \varphi_{2}\left(\varphi_{1}\left(d^{\prime}\right)\right)\right)$ holds in $K$, a contradiction.

Part of the proof of our theorem involves showing how to repair all failures in a lattice. Before describing the constructions by which this is accomplished, we make some observations.

Denote the lattice of ideals of a lattice $L$ by $\mathscr{J}(L)$. Let $L$ and $K$ be lattices and let $\phi$ be a homomorphism of $L$ onto $K$. For $I \in \mathscr{F}(K)$, consider the set

$$
\varphi^{-1}(I)=\{x \in L \mid \varphi(x) \in I\} .
$$

$\mathscr{P}^{-1}(I)$ is an ideal of $L$, and hence $\varphi^{-1}$ is a map of $\mathscr{F}(K)$ into $\mathscr{F}(L)$ 
which is easily seen to be order preserving and one-to-one. Moreover, since meets of ideals are defined by set intersection, $\varphi^{-1}$ is also meet preserving.

LEMmA 4. The $\operatorname{map} \varphi^{-1}: \mathscr{F}(K) \rightarrow \mathscr{F}(L)$ is an embedding if and only if $\varphi$ satisfies the condition

(*) if $y \in L, x_{1}, x_{2} \in K$, and $\varphi(y) \leqq x_{1} \vee x_{2}$, then $y \leqq y_{1} \vee y_{2}$ for some $y_{1}, y_{2} \in L$ satisfying $\varphi\left(y_{1}\right)=x_{1}, \varphi\left(y_{2}\right)=x_{2}$.

Proof. To prove the "if" direction, by the above remarks we need only show that for $I, J \in \mathscr{I}(K), \varphi^{-1}(I \vee J) \subseteq \varphi^{-1}(I) \vee \varphi^{-1}(J)$. Let $x \in \varphi^{-1}(I \vee J)$; then $\varphi(x) \in I \vee J$, so there exist $x_{1} \in I, x_{2} \in J$ such that $\varphi(x) \leqq x_{1} \vee x_{2}$. By $\left(^{*}\right)$ there are $y_{1}, y_{2} \in L$ such that $\varphi\left(y_{1}\right)=x_{1}$, $\varphi\left(y_{2}\right)=x_{2}$, and $x \leqq y_{1} \vee y_{2}$. But then $y_{1} \in \varphi^{-1}(I), \quad y_{2} \in \varphi^{-1}(J)$, so $x \in \varphi^{-1}(I) \vee \varphi^{-1}(J)$, as desired.

Conversely, suppose that $\varphi^{-1}$ is an embedding, and let $y \in L$ and $x_{1}, x_{2} \in K$ be such that $\varphi(y) \leqq x_{1} \vee x_{2}$. Then $(\varphi(y)] \leqq\left(x_{1}\right] \vee\left(x_{2}\right]$ in $\mathscr{J}(K)$, and since $\varphi^{-1}$ is join preserving we have that $y \in \mathscr{P}^{-1}((\varphi(y)]) \subseteq$ $\varphi^{-1}\left(\left(x_{1}\right]\right) \vee \varphi^{-1}\left(\left(x_{2}\right]\right)$. Thus there exist $y_{1} \in \varphi^{-1}\left(\left(x_{1}\right]\right), y_{2} \in \varphi^{-1}\left(\left(x_{2}\right]\right)$ such that $y \leqq y_{1} \vee y_{2}$. Clearly we may assume that $\varphi\left(y_{1}\right)=x_{1}$ and $\varphi\left(y_{2}\right)=x_{2}$.

The next three propositions allow us to repair all failures in a lattice. The constructions used in these results are slight modifications of constructions that have appeared elsewhere; that of Proposition 5 is taken from Theorem 4.4 of H. S. Gaskill, G. Grätzer, and C. R. Platt [5], and that of Propositions 6 and 7 is taken from Theorem 3.1 of T. G. Kucera and B. Sands [9]. We have included Figures 1 and 2 to illustrate the constructions in Propositions 5 and 6 respectively.

Proposition 5. Let $\boldsymbol{x}=\langle a, b, c, d\rangle$ be a failure of $\left(W_{l}\right)$ or $\left(W_{u}\right)$ in the lattice $L$. There exists a lattice $L_{x}$ and a homomorphism $\varphi_{x}$ of $L_{x}$ onto $L$ satisfying $\left(^{*}\right)$ such that $\boldsymbol{x}$ is repaired in $L_{x}$ by $\varphi_{x}$.

REMARK. One method of repairing failures of $\left(W_{l}\right)$ or $\left(W_{u}\right)$ is already in the literature; namely, the "interval construction" of A. Day [3]. However, it will be crucial for the proof of our main theorem that the homomorphisms we use to repair failures satisfy $\left.{ }^{*}\right)$, and it is easy to verify that the homomorphism associated with the interval construction does not enjoy this necessary property.

Proof of Proposition 5. Let $Z$ be the integers with their natural order and let $\boldsymbol{E}$ and $\boldsymbol{O}$ denote the sets of even and odd integers, 
respectively. Extend $\boldsymbol{Z}$ to $\boldsymbol{Z}_{b}=\boldsymbol{Z} \cup\{-\infty, \infty\}$ where $-\infty$ is the least and $\infty$ the greatest element of $Z_{b}$. Setting $u=a \wedge b$ and $v=c \vee d$, we define a subset $L_{x}$ of $L \times Z_{b}$ by

$$
\begin{aligned}
L_{x}= & ([b) \times\{\infty\}) \cup((d] \times\{-\infty\}) \cup(((v]-(d]) \times \boldsymbol{E}) \\
& \cup((((L-(v]) \cup[u))-[b)) \times \boldsymbol{O}) .
\end{aligned}
$$

(Figure 1(c) shows $L_{x}$ for the case when $L$ is the lattice of Figure 1(a) and $x=\langle a, b, c, d\rangle$. Figure $1(b)$ shows $L_{x}$ as a subset of $L \times Z_{b}$.) It is not hard to verify that each element of $L \times Z_{b}$ that

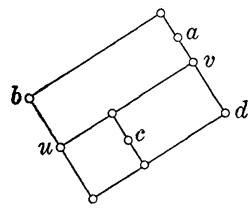

(a) $L$

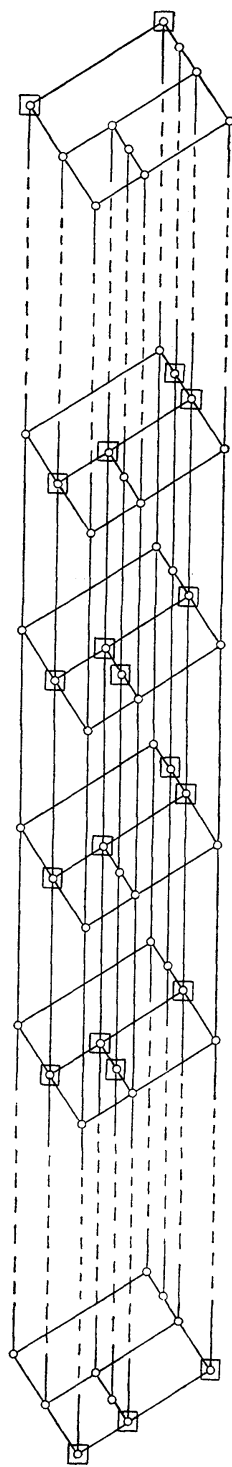

(b) $L \times Z_{b}$

FIGURE 1

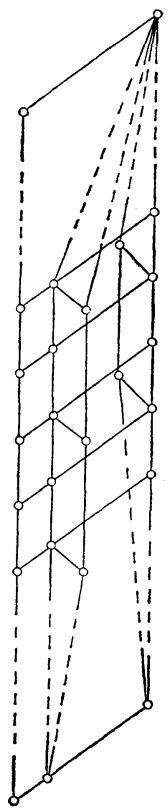

(c) $L_{x}$ 
is not of the form $\langle y,-\infty\rangle$ for $y \not \equiv d$ has a least upper bound in $L_{x}$. This and a dual observation shows that $L_{x}$, with the partial order inherited from $L \times \boldsymbol{Z}_{b}$, is a lattice. Also, the projection $\pi_{1}: L \times \boldsymbol{Z}_{b} \rightarrow L$ restricts to a homomorphism $\varphi_{x}$ of $L_{x}$ onto $L$.

We first claim that $\varphi_{x}$ satisfies $\left(^{*}\right)$. Let $\langle y, t\rangle \in L_{x}$ and $x_{1}, x_{2} \in L$ be such that $y=\varphi_{x}(\langle y, t\rangle) \leqq x_{1} \vee x_{2}$. There exist $i, j \in Z_{b}$ such that $y_{1}=\left\langle x_{1}, i\right\rangle$ and $y_{2}=\left\langle x_{2}, j\right\rangle$ are in $L_{x}$. If $y \leqq d$ then $t=-\infty$, so $\langle y, t\rangle \leqq y_{1} \vee y_{2}$, as desired. Also, if $x_{1} \vee x_{2} \geqq b$ then $y_{1} \vee y_{2}=$ $\left\langle x_{1} \vee x_{2}, \infty\right\rangle$, whence again $\langle y, t\rangle \leqq y_{1} \vee y_{2}$. Thus we may assume $y \geqq d$ and $x_{1} \vee x_{2} \not b$, and without loss of generality we have both $y$ and $x_{1}$ in $L-((d] \cup[b))$, implying that $t, i \in Z$. Since, for any $x \in L$ and $n \in Z,\langle x, n\rangle \in L_{x}$ implies $\langle x, n \pm 2\rangle \in L_{x}$, we may choose $i \geqq t$, and so $\langle y, t\rangle \in y_{1} \vee y_{2}$ holds in any case, showing that $\varphi_{x}$ satisfies $\left({ }^{*}\right)$.

Secondly, we show that $\boldsymbol{x}$ is repaired in $L_{x}$ by $\varphi_{x}$. Let $a^{\prime}, b^{\prime}, c^{\prime}, d^{\prime} \in L_{x}$ be such that $\varphi_{x}\left(a^{\prime}\right)=a, \varphi_{x}\left(b^{\prime}\right)=b, \varphi_{x}\left(c^{\prime}\right)=c$, and $\varphi_{x}\left(d^{\prime}\right)=d$. It follows that $a^{\prime}=\langle a, i\rangle$ where $i \in \boldsymbol{O}, c^{\prime}=\langle c, j\rangle$ where $j \in E, b^{\prime}=\langle b, \infty\rangle$, and $d^{\prime}=\langle d,-\infty\rangle$. Thus $a^{\prime} \wedge b^{\prime}=\langle a \wedge b, i\rangle$ and $c^{\prime} \vee d^{\prime}=\langle c \vee d, j\rangle$, whence if $a^{\prime} \wedge b^{\prime} \leqq c^{\prime} \vee d^{\prime}$ we have $i \leqq j$. If $\boldsymbol{x}$ is failure of $\left(W_{l}\right)$, assume that $\left\langle a^{\prime}, b^{\prime}, c^{\prime}, d^{\prime}\right\rangle$ is a failure of $\left(W_{l}\right)$ in $L_{x}$; then $c^{\prime} \leqq a^{\prime} \wedge b^{\prime}$, yielding $j \leqq i$ and thus $i=j$, which is impossible since $i$ is odd and $j$ is even. Thus $\left\langle a^{\prime}, b^{\prime}, c^{\prime}, d^{\prime}\right\rangle$ cannot be a failure of $\left(W_{l}\right)$. Similarly, if $\boldsymbol{x}$ is a failure of $\left(W_{u}\right),\left\langle a^{\prime}, b^{\prime}, c^{\prime}, d^{\prime}\right\rangle$ is not a failure of $\left(W_{u}\right)$ in $L_{x}$. Hence $\boldsymbol{x}$ is repaired in $L_{x}$ by $\varphi_{x}$.

Proposition 6. Let $\boldsymbol{x}=\langle a, b, c, d\rangle$ be a failure of $\left(S D_{\vee}\right)$ in the lattice $L$. There exists a lattice $L_{x}$ and a homomorphism $\varphi_{x}$ of $L_{x}$ onto $L$ satisfying $\left({ }^{*}\right)$ such that $\boldsymbol{x}$ is repaired in $L_{x}$ by $\varphi_{*}$.

Proof. Let $\boldsymbol{Z}_{b}, \boldsymbol{E}$, and $\boldsymbol{O}$ be as in Proposition 5, and set $p=a \vee$ $(b \wedge c)$. Define a subset $L_{x}$ of $L \times Z_{b}$ by

$$
\begin{aligned}
L_{x}= & ((p] \times\{-\infty\}) \cup((L-((p] \cup(b])) \times \boldsymbol{E}) \\
& \cup((L-((p] \cup(c])) \times \boldsymbol{O}) .
\end{aligned}
$$

Figure 2(c) shows $L_{x}$ when $L$ in the lattice of $2(a)$ and $\boldsymbol{x}=\langle a, b, c, d\rangle$, and Figure 2(b) shows $L_{x}$ as a subset of $L \times Z_{b}$. It is easy to see that $L_{x}$ is a join-semilattice, and that each element of $L \times \boldsymbol{Z}_{b}$ that is not of the form $\langle y, \infty\rangle$ has a greatest lower bound in $L_{x}$; whence $L_{x}$, with the partial order inherited from $L \times \boldsymbol{Z}_{b}$, is a lattice. Furthermore, the projection $\pi_{1}: L \times Z_{b} \rightarrow L$ again restricts to a homomorphism $\varphi_{x}$ of $L_{x}$ onto $L$.

To show that $\varphi_{x}$ satisfies $\left({ }^{*}\right)$, let $\langle y, t\rangle \in L_{x}$ and $x_{1}, x_{2} \in L$ be such that $y=\varphi_{x}(\langle y, t\rangle) \leqq x_{1} \vee x_{2}$. There exist $i, j \in \boldsymbol{Z}_{b}$ such that $y_{1}=$ $\left\langle x_{1}, i\right\rangle$ and $y_{2}=\left\langle x_{2}, j\right\rangle$ are in $L_{x}$. If $y \leqq p$, then $t=-\infty$, so $\langle y, t\rangle \leqq$ 


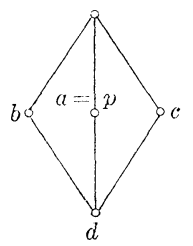

(a) $J$

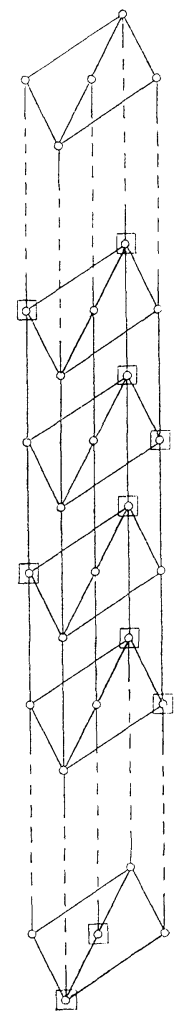

(b) $L \times Z_{b}$

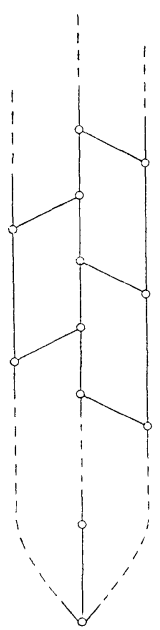

(c) $L$

FIGURE 2

$y_{1} \vee y_{2} . \quad$ On the other hand, if $y \not z p$, then without loss of generality we may let $x_{1} \not \leq p$, and both $t$ and $i$ are in $Z$. We may now choose $i$ such that $\left\langle x_{1}, i\right\rangle \in L_{\mathrm{r}}$ and $t \leqq i$, and thus $\langle y, t\rangle \leqq y_{1} \vee y_{2}$ follows in either case, proving that $\phi_{x}$ satisfies $\left({ }^{*}\right)$.

To show that $\boldsymbol{x}$ is repaired in $L_{x}$ by $\varphi_{x}$, let $a^{\prime}, b^{\prime}, c^{\prime}, d^{\prime} \in L_{x}$ be such that $\varphi_{x}\left(a^{\prime}\right)=a, \varphi_{x}\left(b^{\prime}\right)=b, \varphi_{x}\left(c^{\prime}\right)=c$, and $\varphi_{x}\left(d^{\prime}\right)=d$. Then $b^{\prime}=\langle b, i\rangle$ where $i \in \boldsymbol{O}, c^{\prime}=\langle c, j\rangle$ where $j \in \boldsymbol{E}$, and since $a \leqq p$, $a^{\prime}=\langle a,-\infty\rangle$. Thus $a^{\prime} \vee b^{\prime}=\langle a \vee b, i\rangle$ and $a^{\prime} \vee c^{\prime}=\langle a \vee c, j\rangle$. Since $i$ is odd and $j$ is even, $a^{\prime} \vee b^{\prime} \neq a^{\prime} \vee c^{\prime}$, so $\left\langle a^{\prime}, b^{\prime}, c^{\prime}, d^{\prime}\right\rangle$ is not a failure of $\left(S D_{\vee}\right)$ in $L_{\lambda}$.

Proposition 7. Let $\boldsymbol{x}=\langle a, b, c, d\rangle$ be a failure of $\left(S D_{\wedge}\right)$ in the lattice $L$. There exists a lattice $L_{x}$ and a homomorphism $\varphi_{x}$ of $L_{\mathrm{v}}$ onto $L$ satisfying $\left(^{*}\right)$ such that $\boldsymbol{x}$ is repaired in $L_{\mathrm{r}}$ by $\varphi_{\mathrm{a}}$.

Proof. Let $p=a \wedge(b \vee c)$, and define a subset $L_{\mathrm{v}}$ of $L \times \boldsymbol{Z}_{b}$ by

$$
\begin{aligned}
L_{\imath}= & ([p) \times\{\infty\}) \cup((L-([p) \cup[b))) \times \boldsymbol{E}) \\
& \cup((L-([p) \cup[c))) \times \boldsymbol{O}) .
\end{aligned}
$$


This construction is just the dual of the one in the previous proposition, so $L_{x}$ is a lattice and we have the natural homomorphism $\varphi_{x}$ of $L_{x}$ onto $L$. An argument dual to that in Proposition 6 shows that $\boldsymbol{x}$ is repaired in $L_{x}$ by $\varphi_{x}$, so we need only show that $\varphi_{x}$ satisfies $\left(^{*}\right)$. Let $\langle y, t\rangle \in L_{x}$ and $x_{1}, x_{2} \in L$ be such that $\varphi_{x}(\langle y, t\rangle) \leqq x_{1} \vee x_{2}$. There exist $i, j \in Z_{b}$ such that $y_{1}=\left\langle x_{1}, i\right\rangle$ and $y_{2}=\left\langle x_{2}, j\right\rangle$ are in $L_{x}$. If $x_{1} \vee x_{2} \geqq p$ then $y_{1} \vee y_{2}=\left\langle x_{1}, i\right\rangle \vee\left\langle x_{2}, j\right\rangle=\left\langle x_{1} \vee x_{2}, \infty\right\rangle \geqq\langle y, t\rangle$; therefore, we assume $x_{1} \vee x_{2} \geqq p$, which implies that $t, i, j \in Z$. Now we can choose $i \in Z$ such that $\left\langle x_{1}, i\right\rangle \in L_{x}$ and $i \geqq t$, whence $\langle y, t\rangle \leqq$ $y_{1} \vee y_{2}$ follows.

Before continuing with the proof of the theorem, we recall the following construction.

Definition 8. Let $\left(L_{i} \mid i \in I\right)$ be a family of lattices, let $L$ be a lattice, and let $\varphi_{i}: L_{i} \rightarrow L$ be a lattice homomorphism for each $i \in I$. Form the direct product $\Pi\left(L_{i} \mid i \in I\right)$, and consider the subset

$$
K=\left\{\boldsymbol{x} \in \Pi L_{i} \mid \varphi_{i}(\boldsymbol{x}(i))=\varphi_{j}(\boldsymbol{x}(j)) \text { for all } i, j \in I\right\} \text {. }
$$

Then $K$ is a sublattice of $\Pi L_{i}$, and is called the pullback of the family $\left(\varphi_{i} \mid i \in I\right)$. Letting $\pi_{i}: K \rightarrow L_{i}$ be the restriction of the projection of $\Pi L_{i}$ onto $L_{i}$, we have $\varphi_{i} \circ \pi_{i}=\varphi_{j} \circ \pi_{j}$ for all $i, j \in I$; hence there is a natural homomorphism $\varphi=\varphi_{i} \circ \pi_{i}$ of $K$ into $L$. If $\varphi_{i}$ is onto for all $i \in I$, then $\varphi$ is onto.

Proposition 9. For any lattice $L$, there exists a lattice $L^{*}$ and a homomorphism $\varphi^{*}$ of $L^{*}$ onto $L$ satisfying $\left({ }^{*}\right)$ that repairs all failures in $L$.

Proof. Let $\mathscr{F}(L)$ be the set of all failures in $L$. From Propositions 5, 6, and 7, we obtain a family $\left(L_{x} \mid \boldsymbol{x} \in \mathscr{F}(L)\right)$ of lattices and a family $\left(\varphi_{x}: L_{x} \rightarrow L \mid x \in \mathscr{F}(L)\right)$ of onto homomorphisms satisfying $\left({ }^{*}\right)$ such that for each $\boldsymbol{x} \in \mathscr{F}(L), \boldsymbol{x}$ is repaired in $L_{x}$ by $\varphi_{x}$. Let $L^{*}$ be the pullback of $\left\{\varphi_{x} \mid \boldsymbol{x} \in \mathscr{F}(L)\right\}$ and let $\varphi^{*}$ be the natural homomorphism of $L^{*}$ onto $L$. Then by Lemma $3, \varphi^{*}$ repairs all failures in $L$. To show that $\phi^{*}$ satisfies $\left(^{*}\right)$, let $p \in L^{*}$ and $u, v \in L$ be such that $\varphi^{*}(\boldsymbol{p}) \leqq u \vee v$. Letting $p_{x}$ denote the $\boldsymbol{x}$ th component of $\boldsymbol{p}$, for each $\boldsymbol{x} \in \mathscr{F}(L)$, we have that $\varphi_{x}\left(p_{x}\right)=\varphi^{*}(\boldsymbol{p}) \leqq u \vee v$. For each $\boldsymbol{x} \in \mathscr{F}(L)$, since $\varphi_{x}$ satisfies $\left({ }^{*}\right)$, there exist $u_{x}, v_{x} \in L_{x}$ such that $\varphi_{x}\left(u_{x}\right)=u, \varphi_{x}\left(v_{x}\right)=v$, and $p_{x} \leqq u_{x} \vee v_{x}$ in $L_{x}$. Then the elements $\boldsymbol{u}=\left(u_{x} \mid \boldsymbol{x} \in \mathscr{F}(L)\right)$ and $\boldsymbol{v}=\left(v_{x} \mid \boldsymbol{x} \in \mathscr{F}(L)\right)$ are clearly in $L^{*}$; moreover $\varphi^{*}(\boldsymbol{u})=u, \varphi^{*}(\boldsymbol{v})=v$, and $\boldsymbol{p} \leqq \boldsymbol{u} \vee \boldsymbol{v}$.

Finally, we are in a position to prove our main result. 
THEOREM 10. Every lattice can be embedded in the ideal lattice of a lattice satisfying $\left(S D_{\vee}\right),\left(S D_{\wedge}\right),\left(W_{l}\right)$, and $\left(W_{u}\right)$.

Proof. Let $L$ be a lattice. Set $L_{0}=L$, and inductively let $L_{n+1}=L_{n}^{*}$ and $\varphi_{n}^{*}: L_{n}^{*} \rightarrow L_{n}, n \geqq 0$, be the lattice and homomorphism of Proposition 9. Let $L_{\infty}$ be the inverse limit of the system of lattices $\left(L_{n} \mid n<\omega\right)$ and homomorphisms $\left(\varphi_{n}^{*} \mid n<\omega\right)$, and let $\varphi_{n}$ : $L_{\infty} \rightarrow L_{n}$ be the natural projection for each $n$.

We first claim that $L_{\infty}$ satisfies $\left(S D_{\vee}\right),\left(S D_{\wedge}\right),\left(W_{l}\right)$, and $\left(W_{u}\right)$. Again note that each of these four conditions is expressible in the form $P(x, y, z, w) \Rightarrow Q(x, y, z, w)$, where $P$ and $Q$ are disjunctions of polynomial equations. Let $\langle\boldsymbol{a}, \boldsymbol{b}, \boldsymbol{c}, \boldsymbol{d}\rangle$ be a failure in $L_{\infty}$; then there exist appropriate $P$ and $Q$ such that $P(\boldsymbol{a}, \boldsymbol{b}, \boldsymbol{c}, \boldsymbol{d})$ holds but $Q(\boldsymbol{a}, \boldsymbol{b}, \boldsymbol{c}, \boldsymbol{d})$ fails. It follows that $P\left(\varphi_{n}(\boldsymbol{a}), \varphi_{n}(\boldsymbol{b}), \varphi_{n}(\boldsymbol{c}), \varphi_{n}(\boldsymbol{d})\right)$ holds for all $n \in \omega$, and, since $L_{\infty}$ is a sublattice of $\Pi\left(L_{n} \mid n \in \omega\right), Q\left(\varphi_{m}(\boldsymbol{\alpha}), \varphi_{m}(\boldsymbol{b}), \varphi_{m}(\boldsymbol{c}), \varphi_{m}(\boldsymbol{d})\right)$ fails for some $m \in \omega$. Therefore $\left\langle\varphi_{m}(\boldsymbol{a}), \varphi_{m}(\boldsymbol{b}), \varphi_{m}(\boldsymbol{c}), \varphi_{m}(\boldsymbol{d})\right\rangle$ is a failure in $L_{m}$. But by construction $\left\langle\varphi_{m+1}(\boldsymbol{a}), \varphi_{m+1}(\boldsymbol{b}), \varphi_{m+1}(\boldsymbol{c}), \varphi_{m+1}(\boldsymbol{d})\right\rangle$ is not a failure in $L_{m+1}$, which contradicts Lemma 3 . Thus there can be no failures in $L_{\infty}$; that is, $L_{\infty}$ satisfies $\left(S D_{\vee}\right),\left(S D_{\wedge}\right),\left(W_{l}\right)$, and $\left(W_{u}\right)$.

Next we prove that the homomorphism $\varphi_{0}$ of $L_{\infty}$ onto $L$ satisfies $\left(^{*}\right)$. Let $\boldsymbol{x} \in L_{\infty}$ and $u_{0}, v_{0} \in L=L_{0}$ be such that $\varphi_{0}(\boldsymbol{x}) \leqq u_{0} \vee v_{0}$. Then $\varphi_{0}(\boldsymbol{x})=\varphi_{0}^{*}\left(\varphi_{1}(\boldsymbol{x})\right) \leqq u_{0} \vee v_{0}$, and since $\varphi_{0}^{*}$ satisfies $\left(^{*}\right)$ there exist $u_{1}, v_{1} \in L_{1}$ such that $\varphi_{0}^{*}\left(u_{1}\right)=u_{0}, \varphi_{0}^{*}\left(v_{1}\right)=v_{0}$, and $\varphi_{1}(\boldsymbol{x}) \leqq u_{1} \vee v_{1}$. Proceeding by induction, assume that we have $u_{n}, v_{n} \in L_{n}$ such that $\varphi_{n}(\boldsymbol{x}) \leqq u_{n} \vee v_{n}$. Then $\varphi_{n}(\boldsymbol{x})=\varphi_{n}^{*}\left(\varphi_{n+1}(\boldsymbol{x})\right) \leqq u_{n} \vee v_{n}$, and since $\varphi_{n}^{*}$ satisfies $\left(^{*}\right)$ there exist $u_{n+1}, v_{n+1} \in L_{n+1}$ such that $\varphi_{n}^{*}\left(u_{n+1}\right)=u_{n}$, $\varphi_{n}^{*}\left(v_{n+1}\right)=v_{n}$, and $\varphi_{n+1}(\boldsymbol{x}) \leqq u_{n+1} \vee v_{n+1}$. Now let $\boldsymbol{u}=\left\langle u_{n}\right| n\langle\omega\rangle$ and $\boldsymbol{v}=\left\langle v_{n}\right| n\langle\omega\rangle$. It follows that $\boldsymbol{u}, \boldsymbol{v} \in L_{\infty}, \varphi_{0}(\boldsymbol{u})=u, \varphi_{0}(\boldsymbol{v})=v$, and $\boldsymbol{x} \leqq \boldsymbol{u} \vee \boldsymbol{v}$, whence $\varphi_{0}$ satisfies $\left({ }^{*}\right)$.

From Lemma $4, \mathscr{J}(L)$ is embedded in $\mathscr{J}\left(L_{\infty}\right)$. Since $L$ is embedded in $\mathscr{I}(L)$, the theorem is proved.

As mentioned earlier, we have the following corollary.

CoROLLARY 11. Every transferable lattice satisfies (W).

REMARK. The use of homomorphisms, pullbacks, and inverse limits to repair failures stems from a proof in a recent paper of A. Day, namely, the proof (see Theorem 3.2 in [4]) that every lattice is a bounded homomorphic image of a lattice satisfying $(W)$.

3. Additional results. In this section we investigate the status of $\mathscr{E}(P)$ for most other combinations $(P)$ of the properties defined 
in the introduction. First, we shall indicate how certain techniques in a paper of G. Grätzer and C. R. Platt [8] can be modified so as to prove that $\mathscr{E}\left(\left(S D_{\vee}\right) \&(S F) \&(X)\right)$ holds.

Let $L$ be a lattice. It has already been observed that there is a lattice $K$ satisfying $(S F)$ such that $L$ is embeddable in $\mathscr{J}(K)$. Hence we need only show that for every lattice $K$ satisfying $(S F)$ there is a lattice $M$ satisfying $\left(S D_{\vee}\right),(S F)$, and $(X)$ such that $\mathscr{J}(K)$ is embeddable in $\mathscr{I}(M)$.

Let $K$ be a lattice satisfying $(S F)$. In [8], Grätzer and Platt construct a lattice $L\left(K_{I}\right)$ satisfying $\left(S D_{\vee}\right)$ such that $K$ can be embedded in $\mathscr{J}\left(L\left(K_{I}\right)\right.$ ). From Lemma 3 and their proof it is clear that they in fact embed $\mathscr{J}(K)$ in $\mathscr{I}\left(L\left(K_{I}\right)\right)$. The lattice $L\left(K_{I}\right)$ consists of certain subsets (called closed subsets) of $K \times \boldsymbol{Z}$, ordered by inclusion.

Now we replace $Z$ by $\omega$, and consider the set $L_{f}\left(K_{I}\right)$ of all finitely generated closed subsets of $K \times \omega$, that is, all closed subsets which are closures of finite subsets of $K \times \omega$. Since $K$ satisfies $(S F)$, each element of $L_{f}\left(K_{I}\right)$ is finite. Hence $L_{f}\left(K_{I}\right)$, ordered by inclusion, is a lattice; in fact $L_{f}\left(K_{I}\right)$ is embeddable in $L\left(K_{I}\right)$ and therefore satisfies $\left(S D_{\vee}\right)$. Furthermore, $L_{f}\left(K_{I}\right)$ is sectionally finite. Next, it can be proved as in [8] that $\mathscr{J}(K)$ is embeddable in $\mathscr{J}\left(L_{f}\left(K_{I}\right)\right.$ ), and moreover the image under this embedding of each ideal in $K$ is a nonprincipal ideal of $L_{f}\left(K_{I}\right)$. Therefore (G. Grätzer [7]) the elements of $L_{f}\left(K_{I}\right)$ may all be "split" to yield a lattice $M$ satisfying $(X)$ such that $\mathscr{J}(K)$ is embeddable in $\mathscr{J}(M)$. It is easy to see that $M$ will still satisfy $\left(S D_{\vee}\right)$ and $(S F)$. Thus we have:

THEOREM 12. $\mathscr{E}\left(\left(S D_{\vee}\right) \&(S F) \&(X)\right)$ holds.

In contrast to the above, we now establish two negative results.

LEMma 13. If a lattice $L$ satisfies $(S F)$ and $\left(S D_{\wedge}\right)$, then $\mathscr{F}(L)$ satisfies $\left(S D_{\wedge}\right)$.

Proof. Let $L$ satisfy $(S F)$ and $\left(S D_{\wedge}\right)$, and let $A, B, C \in \mathscr{J}(L)$ satisfy $A \cap B=A \cap C$. Let $p \in A \cap(B \vee C)$. There exist $b \in B$ and $c \in C$ such that $p \leqq b \vee c$. By $(S F)$, there exist largest elements $b_{0} \in B$ and $c_{0} \in C$ such that $b_{0}, c_{0} \leqq b \vee c$. Since $p \in A, p \wedge b_{0} \in A \cap B$ and $p \wedge c_{0} \in A \cap C=A \cap B$. Thus the element $q=\left(p \wedge b_{0}\right) \vee$ $\left(p \wedge c_{0}\right) \in A \cap B$, and by the choice of $b_{0}, p \wedge c_{0} \leqq q \leqq b_{0}$. Hence $p \wedge c_{0} \leqq p \wedge b_{0}$; by symmetry we have that $p \wedge b_{0}=p \wedge c_{0}$. Since $L$ satisfies $\left(S D_{\wedge}\right), p \wedge b_{0}=p \wedge\left(b_{0} \vee c_{0}\right)=p \wedge(b \vee c)=p$. We conclude that $p \in A \cap B$, and so $A \cap(B \vee C)=A \cap B$, showing that $\mathscr{J}(L)$ satisfies $\left(S D_{\wedge}\right)$. 
CoRollary 14. $\mathscr{E}\left((S F) \&\left(S D_{\wedge}\right)\right)$ fails.

LEMMA 15. If a lattice $L$ satisfies $(S F)$ and $\left(W_{u}\right)$, then $\mathscr{J}(L)$ satisfies $\left(W_{u}\right)$.

Proof. Let $L$ satisfy $(S F)$ and $\left(W_{u}\right)$, and suppose that $\langle A, B, C, D\rangle$ is a $\left(W_{u}\right)$-failure in $\mathscr{F}(L)$. Then there exists an element $x \in A \cap B$ such that $x \notin C$ and $x \notin D$, and an element $b \in B$ such that $b \geqq x$ and $b \notin C \vee D$. Since $L$ satisfies $(S F)$, there exists a largest element $x_{0} \in A \cap B$ such that $x_{0} \leqq b$; note that $x_{0} \geqq x$ and so $x_{0} \notin C, x_{0} \notin D$. Since $x_{0} \in A \cap B \subseteq C \vee D$, there exist $c \in C, d \in D$ such that $x_{0} \leqq c \vee d$. However, $b \notin C \vee D$, so $b \not c \vee d$. Finally, since $A \supset C \vee D$, we may choose $a \in A$ such that $a>c \vee d$. But now $a \wedge b \geqq(c \vee d) \wedge b \geqq x_{0}$, and by the maximality of $x_{0}$ we obtain that $a \wedge b=x_{0}$. Hence the quadruple $\langle a, b, c, d\rangle$ is a failure of $\left(W_{u}\right)$ in $L$, contradicting the hypothesis. We conclude that $\mathscr{F}(L)$ satisfies $\left(W_{u}\right)$.

CoROllary 16. $\mathscr{E}\left((S F) \&\left(W_{u}\right)\right)$ fails.

To end this paper we ask two questions that are still open:

(i) Does $\mathscr{E}\left((S F) \&\left(W_{l}\right)\right)$ hold?

(ii) Does $\mathscr{E}\left((S F) \&\left(W_{l}\right) \&\left(S D_{\vee}\right)\right)$ hold?

\section{REFERENCES}

1. R. Antonius and I. Rival, A note on Whitman's property for free lattices, Algebra Universalis, 4 (1974), 271-272.

2. K. A. Baker and A. W. Hales, From a lattice to its ideal lattice, Algebra Universalis, 4 (1974), 250-258.

3. A. Day, A simple solution to the word problem for lattices, Canad. Math. Bull., 13 (1970), 253-254.

4. - Splitting lattices generate all lattices, Algebra Universalis, 7 (1977), 163-169.

5. H. S. Gaskill, G. Grätzer, and C. R. Platt, Sharply transferable lattices, Canad. J. Math., 27 (1975), 1247-1262.

6. G. Grätzer, Universal Algebra in Trends in Lattice Theory, 173-215. New York, Van Nostrand Reinhold, 1970.

7. - A property of transferable lattices, Proc. Amer. Math. Soc., 43 (1974), 269-271.

8. G. Grätzer and C. R. Platt, Two embedding theorems for lattices, Proc. Amer. Math. Soc., 69 (1978), 21-24.

9. T. G. Kucera and B. Sands, Lattices of lattice homomorphisms, Algebra Universalis. 8 (1978), 180-190.

10. P. M. Whitman, Lattices, equivalence relations, and subgroups. Bull. Amer. Math. Soc., 52 (1946), 507-522.

Received May 3, 1978 and in revised form December 29, 1978. The work of all three authors was supported by the National Research Council of Canada.

UNIVERSITY OF MANITOBA

Winnipeg, Manitoba, Canada 



\section{PACIFIC JOURNAL OF MATHEMATICS}

\section{EDITORS}

DONALD BABBITT (Managing Editor)

University of California

Los Angeles, California 90024

HUgo RossI

University of Utah

Salt Lake City, UT 84112

C. C. MOORE and ANDREW OGG

University of California

Berkeley, CA 94720

\section{J. DUGUNDJI}

Department of Mathematics University of Southern California Los Angeles, California 90007

R. Finn and J. Milgram Stanford University Stanford, California 94305

\section{ASSOCIATE EDITORS}

E. F. BECKENBACH

B. H. NEUMANN

F. WOLF

K. YoshidA

\section{SUPPORTING INSTITUTIONS}

UNIVERSITY OF BRITISH COLUMBIA CALIFORNIA INSTITUTE OF TECHNOLOGY UNIVERSITY OF CALIFORNIA MONTANA STATE UNIVERSITY UNIVERSITY OF NEVADA, RENO NEW MEXICO STATE UNIVERSITY OREGON STATE UNIVERSITY UNIVERSITY OF OREGON
UNIVERSITY OF SOUTHERN CALIFORNIA STANFORD UNIVERSITY UNIVERSITY OF HAWAII UNIVERSITY OF TOKYO UNIVERSITY OF UTAH WASHINGTON STATE UNIVERSITY UNIVERSITY OF WASHINGTON 


\section{Pacific Journal of Mathematics}

Vol. 85, No. $1 \quad$ September, 1979

Ralph Alexander, Metric averaging in Euclidean and Hilbert spaces...... 1

B. Aupetit, Une généralisation du théorème de Gleason-Kahane-Żelazko

pour les algèbres de Banach..............................

Lung O. Chung, Jiang Luh and Anthony N. Richoux, Derivations and

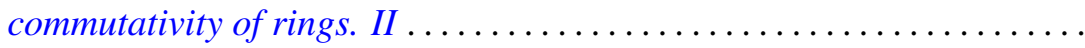

Lynn Harry Erbe, Integral comparison theorems for third order linear

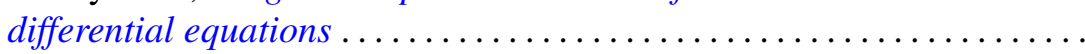

Robert William Gilmer, Jr. and Raymond Heitmann, The group of units of a

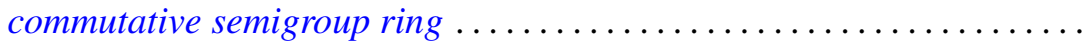

George Grätzer, Craig Robert Platt and George William Sands, Embedding lattices into lattices of ideals ...........................

Raymond D. Holmes and Anthony Charles Thompson, $n$-dimensional area and content in Minkowski spaces ....................... 77

Harvey Bayard Keynes and M. Sears, Modelling expansion in real flows....

Taw Pin Lim, Some classes of rings with involution satisfying the standard

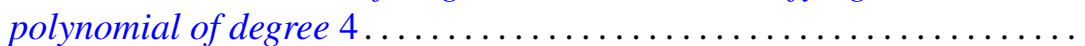

Garr S. Lystad and Albert Robert Stralka, Semilattices having bialgebraic congruence lattices ................................... 131

Theodore Mitchell, Invariant means and analytic actions . . . . . . . . . 145

Daniel M. Oberlin, Translation-invariant operators of weak type ........ 155

Raymond Moos Redheffer and Wolfgang V. Walter, Inequalities involving

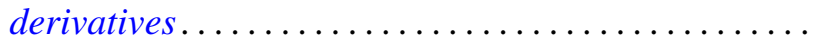

Eric Schechter, Stability conditions for nonlinear products and semigroups ................................

Jan Søreng, Symmetric shift registers ........................ 201

Toshiji Terada, On spaces whose Stone-Čech compactification is $\mathrm{Oz} \ldots \ldots .231$

Richard Vrem, Harmonic analysis on compact hypergroups ... 